\title{
NUMERICAL COMPARISON OF DRAG MODELS APPLIED TO SUBSEA GAS DISPERSION
}

\author{
AMARANATH S. KUMARA \& TAEWOOK KIM \\ University of South-Eastern Norway, Norway
}

\begin{abstract}
Subsea gas releases can have catastrophic impacts on human life, offshore assets, and the environment. As a result of major accidents that occurred recently, government regulations and company policies enforce a formal assessment of risks related to subsea gas releases. The main objective of subsea gas dispersion modelling is to predict the properties such as plume width, gas volume fraction and mean velocities at the sea surface in order to provide input data for risk models quantifying the topside risk exposure on offshore installations. This requires a comprehensive understanding of the dynamics of underwater releases of natural gas. This paper presents a comparison of different drag models applied for subsea gas dispersion modelling. ANSYS Fluent is used as the Computational Fluid Dynamics (CFD) modelling framework of the subsea gas plume hydrodynamics, while the changes of bubble's density and size is included as an external user defined functions (UDFs) hooked to the Fluent's main code structure. Four different drag models are compared, namely spherical drag law, modified spherical drag law, Xia's drag law and Tomiyama's drag law. The drag models are also incorporated into the main code structure as external UDFs. A combination of the two methods - Eulerian-Eulerian and Lagrangian - is used to model the bubbling behaviour of the subsea gas dispersion. The predicted results are validated against the experimental data presented by Engebretsen back in 1997. It is observed that the drag model in the CFD simulations seems to be a factor that could affect underwater plume physics. The predicted results show that the drag models including bubble shape show better agreement than the ones without including bubble shape in general.
\end{abstract}

Keywords: subsea gas dispersion, drag model, computational fluid dynamics, gas plume, bubble behaviour, discrete phase model, volume of fluid model.

\section{INTRODUCTION}

The physics of subsea gas dispersion has been an interesting research area for almost a century. A comprehensive review of the current status of the modelling of subsea gas releases has been presented by Olsen and Skjetne [1] and Rew et al. [2]. The type of modelling available for subsea gas dispersion predictions ranges from simple empirical approximation to integral or Computational Fluid Dynamics (CFD) computer programs. The empirical model is the simplest one which assumes that the plume radius is proportional to the release depth or correlations. On the other hand, integral type models are based on local similarity. For instance, a velocity profile is assumed to have a similar form at different elevations. The plume properties can be well described by Gaussian profiles. The entrainment of water into the gas plume is modelled using entrainment coefficients. The most complex models are represented by CFD or field codes by solving Navier-Stokes Equations. The advantage of CFD models over the integral models is that CFD models do not require the use of empirical constants. However, CFD programs are computationally expensive than integral models, and simulation time may be a matter of days or weeks rather than minutes [3]. A survey of operators showed that empirical models are used extensively within the risk assessment studies, due to their ease of use for large number of scenarios, although CFD has been used in a research context [2]. In recent industrial applications, the speed and simplicity of integral models make them a more viable option, and due to the large uncertainties related to gas dissolution and plume behaviour at large depths there is no guarantee that the more complex 
CFD programs will yield better results than integral models. In all cases, lack of full-scale data has meant that the models have not been validated for the high release rates common for blowout ands or the rupture of subsea pipelines.

Three different multiphase CFD methods were evaluated to assess their applicability to subsea gas dispersion modelling by Cloete et al. [4]. These models include the EulerianEulerian multi-fluid approach, the Eulerian-Eulerian mixture model and also a combined model consisting of the Eulerian-Lagrangian discrete phase model (DPM) and the EulerianEulerian volume of fluid (VOF) model. They concluded that pure Eulerian-Eulerian approaches were computationally expensive, and the free surface was poorly modelled as these models are not designed for tracking the sharp interfaces between multiphase flow systems. However, VOF variant of Eulerian-Eulerian multiphase model is specifically programmed for the tracking of sharp interfaces between various phases and can therefore give a good representation of the free surface interaction of subsea gas plumes [4].

The VOF model with sharp interface tracking has a limited applicability in modelling subsea gas dispersion process. The model seeks to track the free interface around every bubble in the rising gas plume hence demands a prohibitively fine grid resolution. On the other hand, the bubble plume behaviour could be predicted by discrete phase model (DPM), where bubbles are essentially treated as point sources of momentum rising through the domain. Cloete et al. [4] concluded that DPM approach is computationally efficient and also very accurate, provided that the force balance over each 'bubble' is specified correctly. Hence, coupled DPM and VOF model was successfully applied for quantitative analysis of subsea gas releases [4].

Cloete et al. [4] has developed a CFD model to predict subsea gas dispersion and free surface behaviour of gas release from subsea pipeline. The multiphase flow behaviour was modelled using the coupled VOF and DPM approach. The turbulence effects were modelled using the standard $k-\epsilon$ model. The model was successfully validated against a wide range of experimental measurements presented by Engebretsen et al. [5] using a $7 \mathrm{~m}$ deep test basin set-up. Pan and Johansen [6] further developed Cloete et al. [4] model by including an enhanced $k-\epsilon$ model which can predict low gas release rates and surface velocities accurately. Wu et al. [7] claimed that both Cloete et al. [4] and Pan and Johansen [6] models were not capable of modelling the transient random wandering behaviour of the rising gas plume. They observed that random wandering behaviour of the subsea gas plume was not correctly modelled by $k-\epsilon$ turbulence model due to the inherent Reynolds-Averaged Navier-Stokes (RANS) nature of the approach. This leads to an over-prediction of the plume central line velocity and an under-prediction of the plume width as there was no mechanism to distribute and dissipate the high momentum gained during the initial gas release phase. The predictions obtained using the large eddy simulation (LES) approach show the inherently random wandering behaviour of the gas plume and both centreline velocity and velocity profile are in much better agreement with the experimental data [7].

Bakli [8] presented a CFD model for predicting deep water multiphase (oil and gas) releases. The fundamental theoretical framework and model set-up was based on model developed by Cloete [9], Cloete et al. [4], Skjetne and Olsen [10], and generalized to allow for the presence of oil droplets and plume tracking, underwater currents and gas dissolution effect. The model was successfully validated against experimental data of Engebretsen et al. [5], Johansen et al. [11] and relevant modelling results provided by the DeepBlow model [8]. The overall predictions presented by Bakli [8] are found to yield quite good agreement with the experiment data.

In the present study, the effect of drag models on subsea gas dispersion process is evaluated. ANSYS Fluent is used as the CFD modelling framework of the subsea gas plume 
hydrodynamics, while the changes of bubble's density and size is included as an external user defined functions (UDFs) hooked to the Fluent's main code structure. These user defined functions are based on the model developed by Bakli [8]. Four different drag models are compared, namely spherical drag law, Modified Spherical Drag law, Xia's drag law and Tomiyama's drag law. The drag models are also incorporated to the main CFD code as external user defined functions (UDFs). The model predictions are validated against the experimental data presented by Engebretsen et al. [5].

\section{MODEL DESCRIPTION}

The model is based on the Eulerian-Lagrangian modelling concept that has been developed to study subsea gas release [4], [8]-[10]. As shown in Fig. 1, the model has two Eulerian phases with interface tracking of free surface separating the ocean and atmosphere. In this case Eulerian mixture model is used. The dispersed bubbles are tracked using the Lagrangian approach. The Eulerian method with interface tracking is a volume of fluid (VOF) method, and the Lagrangian tracking method is a discrete phase model (DPM). This is also known as a coupled DPM-VOF model [4].

\subsection{The coupled DPM and VOF model}

The continuous phases, i.e. air above the free surface and water below the free surface, is mathematically described by the VOF model which is a standard single-fluid EulerianEulerian mixture model where a single set of governing equations is shared between different phases [4]. The VOF model is used for accurate tracking of the free surface separating water and air. The VOF model solves for conservation of mass

$$
\frac{\partial}{\partial t}\left(\alpha_{q} \rho_{q}\right)+\nabla \cdot\left(\alpha_{q} \rho_{q} \vec{v}_{q}\right)=0
$$

and momentum

$$
\frac{\partial}{\partial \mathrm{t}}(\rho \overrightarrow{\mathrm{v}})+\nabla(\rho \overrightarrow{\mathrm{v}} \overrightarrow{\mathrm{v}})=-\nabla \mathrm{p}+\nabla \cdot\left[\mu\left(\nabla \overrightarrow{\mathrm{v}}+\nabla \overrightarrow{\mathrm{v}}^{\mathrm{T}}\right)\right]+\rho \overrightarrow{\mathrm{g}}+\overrightarrow{\mathrm{F}}
$$

The momentum contribution from the gas bubbles are provided as input to the external force $\vec{F}$. The mixture density is estimated based on the phase densities as given below

$$
\rho=\sum \alpha_{q} \rho_{q}
$$

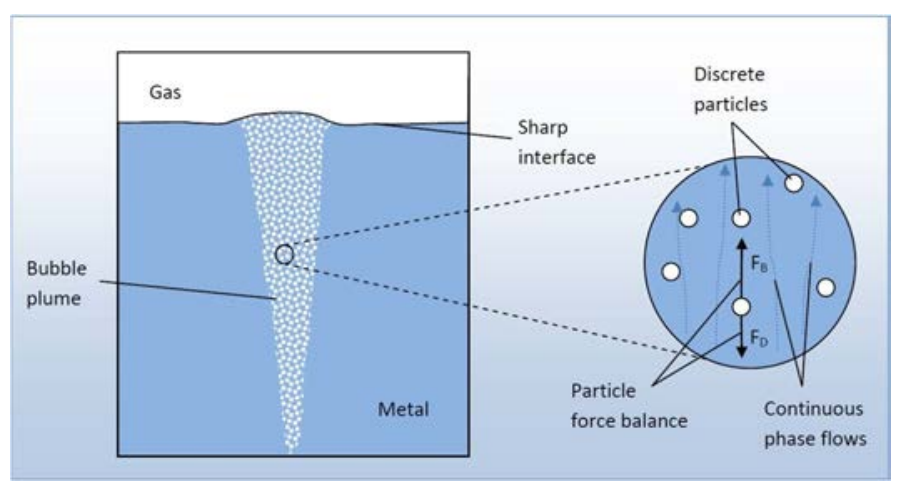

Figure 1: Sketch of the combined Eulerian-Eulerian-Lagrangian approach [8]. 
The viscosity is modelled as the sum of turbulent viscosity and molecular mixture viscosity.

$$
\mu=\mu_{T+} \mu_{M},
$$

where the molecular mixture viscosity is given by the properties of the phases equivalent to the mixture density and the turbulent viscosity is given by the turbulence model. The turbulence effects are modelled using the standard $k-\epsilon$ turbulence model with default model coefficients [12]. In addition to solving the continuity, momentum and turbulence equations, the VOF model also performs procedures for tracking the interface between the phases. In this case, VOF model surface-tracking scheme, named Geo-Reconstruction is used for transient tracking of interface of water and atmospheric air.

The DPM is used for tracking the bubbles in the gas plume. The DPM performs Lagrangian trajectory calculations for the dispersed phase, including coupling with the continuous phase [8]. The DPM is a highly efficient way of tracking the bubble plume of dilute and moderately dense plumes. It should be noted that the DPM model assumes that particles do not occupy any volume in the computational domain. Therefore, the DPM model should carefully applied for tracking gas bubbles at higher gas fractions. Cloete et al. [4] concluded that this assumption is acceptable in the bulk of the plume for low to medium gas rates but is violated in the lower plume regions when higher gas flow rates are implemented [4].

The DPM tracks discrete particles through the domain in the Lagrangian sense by implementing a force balance over each particle as given in eqn (5):

$$
\frac{\mathrm{d} \vec{u}_{p}}{d t}=F_{D}\left(\vec{u}-\vec{u}_{p}\right)+\frac{\vec{g}\left(\rho_{p}-\rho\right)}{\rho_{p}}+\vec{F}_{p}
$$

This force balance accounts for the particle inertia with the forces acting on the particle. On the right side, the first term is the drag force per unit particle mass, second term is a gravity term and third term is an additional acceleration. In the current problem set-up additional forces such as lift were assumed to be negligible. As shown in eqn (5), the particle motion is influenced by the velocity of the continuous phases since the velocity, $u$, of these phases are present in the drag term.

\subsection{Drag models}

Four different drag models are compared, namely spherical drag law, modified spherical drag law, Xia's drag law and Tomiyama's drag law applied to subsea gas dispersion. The different drag models are discussed below. The drag models are included in the main CFD code either by basic drag laws that Fluent provides, or as user-defined functions (UDFs).

\subsubsection{Spherical drag law}

This drag law applies to particles of smooth spherical shape. The conventional correlation for the drag on a sphere in steady motion is presented as a graph, called the 'standard drag curve' [13]. It shows the relationship between drag coefficient $\left(C_{d}\right)$ and Relative Reynolds number $(R e)$. The drag coefficient, for smooth particles can be taken from

$$
\mathrm{C}_{d}=a_{1}+\frac{a_{2}}{R e}+\frac{a_{3}}{R e^{3}}
$$

where $a_{1}, a_{2}$ and $a_{3}$ are constants that apply over several ranges of $R e$ given by Morsi and Alexander [13]. The value of the drag coefficient varies widely with Reynolds number. At low Reynolds number, eqn (6) becomes the creeping flow result, $\mathrm{C}_{d}=24 / R e$ while at the 
highest Reynolds numbers, eqn (6) plateaus at $C_{d} \approx 0.44$. The spherical drag model is available in ANSYS Fluent.

\subsubsection{Modified spherical drag law}

This modified spherical drag law is also based on the drag curve developed by Clift et al. [14]. A number of empirical correlations have been proposed to approximate the drag curve. The modified spherical drag law is applicable for high Reynolds number compared to the standard spherical drag law. The modified spherical drag law is coded as a UDF in the CFD model.

\subsubsection{Xia's drag law}

The shape of bubble has significant influence on drag force acting on rising bubble. Accordingly, the drag law of Xia et al. [15] is applied to account for larger bubbles that are deformed from the standard spherical shape:

$$
\mathrm{C}_{d}=\frac{2}{3}\left(\frac{E_{0}}{3}\right)^{0.5} \text {. }
$$

The Eotvos number, $E_{0}$ is a dimensionless number that describe the characteristic shape of the bubble and is expressed as a function of the liquid and gas densities, the bubble diameter and surface or interfacial tension:

$$
E_{0}=\frac{g\left(\rho_{l}-\rho_{g}\right) d_{b}^{2}}{\sigma}
$$

The drag law presented by Xia et al. [15] has been validated against the experimental data using a liquid metal. The bubbles in a liquid metal rather have large sizes and distorted shape in the turbulent region. Hence, Xia's drag law is applicable for larger bubbles that are significantly deformed from the spherical shape. The Xia's drag law has been implemented in CFD subsea gas dispersion models presented by Cloete et al. [4] and Bakli [8]. The model is included in the main code structure as an external UDFs hooked to the Fluent's main code structure.

\subsubsection{Tomiyama's drag law}

Tomiyama's model is well suited to gas-liquid flows in which the bubbles can have a range of shapes [16]. The drag closure model has been derived experimentally in terms of $R e$ and $E_{0}$ as given below:

$$
\mathrm{C}_{d}=\max \left(\min \left(\frac{24}{R e}\left(1+0.15 R e^{0.687}\right), \frac{72}{R e}\right), \frac{8}{3} \frac{E_{0}}{E_{0}+4}\right) .
$$

The Tomiyama's drag model is incorporated into the CFD code as an external UDF.

\subsection{Turbulence model}

The standard $k-\epsilon$ model with default model coefficients [12] is used for modelling the turbulence effects in the surrounding water. The velocity fluctuations in the turbulent flow filed leads to an additional drag force which is not accounted for by the drag term. The turbulent dispersion of the particles is implemented using a stochastic tracking (random walk) approach similar to the CFD models presented by Cloete et al. [4] and Bakli [8]. In this case, the trajectory of the particle is influenced by the instantaneous fluid velocity, $\bar{u}+u^{\prime}$, and not solely by the Reynolds averaged velocity, $\bar{u}$. The fluctuating component that prevails during 
the lifetime of the eddy is determined by eqn (10), assuming that a Gaussian probability distribution is followed.

$$
u^{\prime}=\zeta \sqrt{\overline{u^{\prime 2}}} \text {. }
$$

Here, the root mean square value of the local fluctuations is multiplied by a normally distributed random number to give the instantaneous fluctuations. The amount of turbulent dispersion is governed by the time spent by a particle inside the turbulent eddy. An integral time scale is used for this purpose [4]:

$$
T_{L}=C_{L} \frac{k}{\varepsilon}
$$

A value of 0.15 is recommended for the Lagrangian time scale constant $\left(C_{L}\right)$ when the standard $k-\epsilon$ model is used.

\subsection{Bubble size model}

As seen from eqn (8), Eotvos number characterizes the bubble shape, and it depends on the bubble diameter. Hence, the drag force of a rising bubble is influenced by the bubble diameter. In addition, the amount of mass transferred from a rising bubble to the surrounding water, is directly dependent on the bubble surface area. The bubble size is determined by turbulence factors and loss of hydrostatic pressure, which affects the material properties of a buoyant bubble. A bubble size model is implemented to account for the effect of variation in bubble diameters accounting for breakup and coalescence mechanisms similar to the CFD model presented by Bakli [8]. The fundamental theory is based on the work of Laux and Johansen [17], and additional modifications are provided by Cloete et al. [4] and Pan [18]. The local mean bubble diameter, $d_{b}$, is given by a transport equation which accounts for loss of bubbles to downstream cells, gain of bubbles from upstream cells, break-up and coalescence. The transport equation for local mean bubble diameter is given below for the Lagrangian framework.

$$
\frac{\partial \rho_{b} d_{b}}{\partial t}=\rho_{b} \frac{d_{b}^{e q}-d_{b}}{\tau_{r e l}}
$$

where $\rho_{b}$ is the bubble bulk density. The relaxation time $\tau_{r e l}$ is controlled by the speed of breakup or the coalescence process, and $d_{b}^{e q}$ is the mean equilibrium diameter. The equilibrium bubble diameter can be defined as the diameter a bubble achieves if it resides sufficiently long time at the same flow conditions. The term at the right hand side forces the local mean bubble diameter towards its equilibrium diameter during a time frame given by the relaxation time [4]. The equilibrium diameter is calculated as follows:

$$
d_{b}^{e q}=C_{1} \phi_{p}^{0.5} \frac{(\sigma / \rho)^{0.6}}{\varepsilon^{0.4}}\left(\frac{\mu_{p}}{\mu}\right)^{0.25}+C_{2}
$$

where $\sigma$ is the surface tension, $\rho$ is the density of the primary phase (water), $\varepsilon$ is the turbulent dissipation energy and $\mu$ denotes the viscosities (subscript $\mathrm{b}$ for the bubbly phase). The coefficients $C_{1}$ and $C_{2}$ are tuning parameters. $C_{2}$ is often taken as the smallest possible bubble size, while $C_{2}$ is the more significant of the two parameters for which most of the tuning procedure evolves around. For air-water systems the following is often used [8]: $C_{1}=4.0$ and $C_{2}=100 \mu \mathrm{m}$. 
The relaxation time is the time that is needed for bubble to reach the equilibrium diameter. The mean bubble diameter will be driven to its equilibrium diameter during a timeframe given by the relaxation time. The relaxation times for breakup $\left(\tau_{b r}\right)$ and coalescence $\left(\tau_{c o}\right)$ are given by the turbulence dissipation rate $(\varepsilon)$ and kinetic energy $(k)$, respectively [8].

$$
\begin{gathered}
\tau_{b r}=d_{p}^{\frac{2}{3}} \varepsilon^{-\frac{1}{3}}, \\
\tau_{c o}=\frac{d_{p}}{0.2 \cdot 6 \cdot \sqrt{\phi_{p} k}} .
\end{gathered}
$$

When the model is implemented in code, if a bubble is bigger than $d_{b}^{e q}$ then the bubble's breakup occurs otherwise the coalescence will occur. The relaxation time is restricted by turbulent microscale that represents the smallest timescale in turbulent flow. Bubble size is restricted to have a diameter size above $0.0001 \mathrm{~m}$ and the fraction of the bubble is also restricted to be below $1.0 e^{-06}$. The bubble size model is also incorporated as an external user defined function (UDF).

\subsection{Model validation}

The CFD model predictions are validated against the subsea gas dispersion experiments performed by Engebretsen et al. [5]. The objective of the experiments was to investigate the phenomena of the gas plume and the gas release above the water surface. The experiments were conducted in a rectangular basin with a depth of $7 \mathrm{~m}$ and a surface area of $6 \times 9 \mathrm{~m}^{2}$. The basin was filled with water and air was released at the bottom at gas rates of 83, 170 and $750 \mathrm{Nl} / \mathrm{s}$ (equivalent to 50,100 and $450 \mathrm{l} / \mathrm{s}$ referred to the state at the inlet). The inlet was comprised of a release valve with a rapidly acting piston injecting gas vertically with arrangements in front of it to reduce the vertical momentum. Because of this momentum breaker, the fluctuations in the gas flow and the length of the inlet jet were minimized. Pure air was injected when parameters below the water surface were of interest and helium-air mixture was used when gas concentration measurements were to be investigated [5].

\subsection{Computational grid}

The shape of geometry is a box with identical size of the basin used for the experiments $(7 \mathrm{~m} \times 6 \mathrm{~m} \times 9 \mathrm{~m})$ [5]. The geometry was created in ANSYS Design Modeler. The primary mesh was created with a uniform grid size of $20 \mathrm{~cm}$. Then the mesh was refined in the expected plume area by Region Adaption with level 2. The effect of Region Adaption of a cell is to divide the cell into two cells that have the identical grid size to each other. The total number of cells in the computational domain was 1,130,920. Fig. 2 illustrates the exterior boundaries of the computational domain and the mesh on a plane inserted in the middle of the computational domain. The mesh of the plane in the middle of the computational domain shows the refined mesh in the plume area.

In the simulations, gas bubbles were injected as particle streams. Injection type of particles was set to be solid-cone type with $0.17 \mathrm{~m}$ of radius and $13^{\circ}$ of cone angle, positioned $33 \mathrm{~cm}$ above the center of the geometry floor. Particle diameter was set to be $5 \mathrm{~mm}$ as initial bubble diameter. Parcels were injected via 10 injection streamlines. The 'standard parcel release method' was used which injects a single parcel per injection stream per time step. This frequency of injection was found to be sufficient to produce an averaged representation of the plume. 


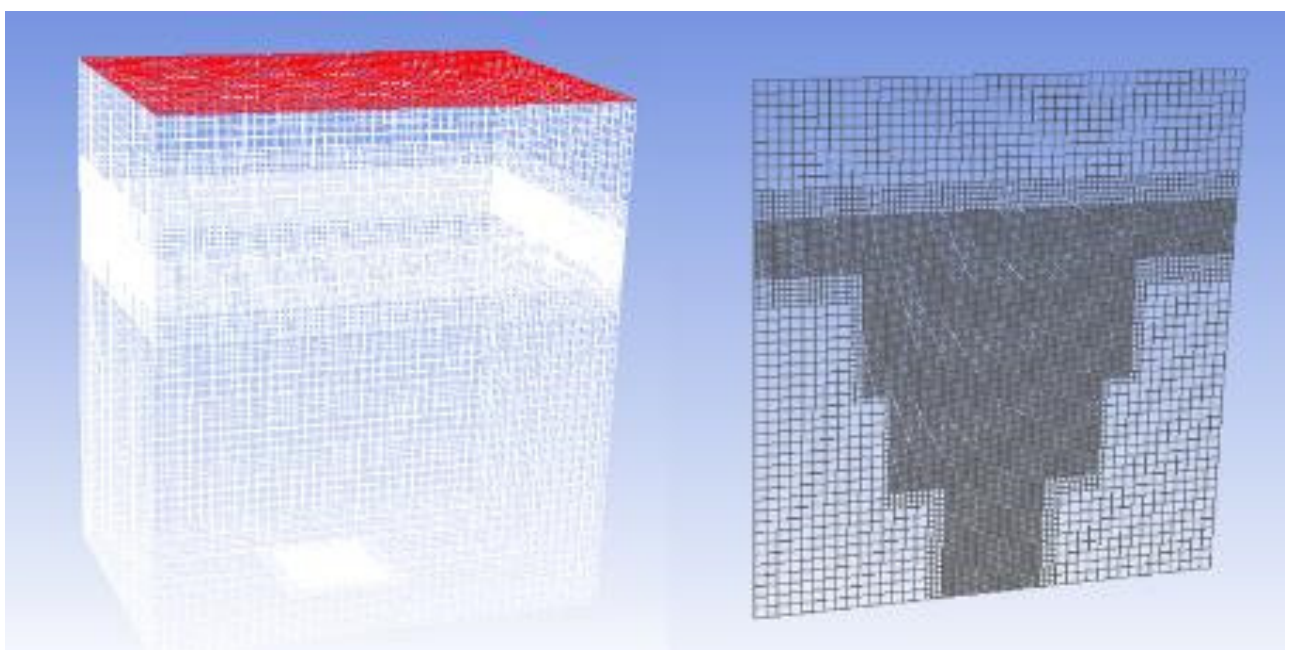

Figure 2: Exterior of the mesh on the left side and the mesh on a plane inserted in the middle on the right side.

\subsection{Numerical implementation and solution procedure}

The computational model described above was implemented in the commercial CFD software ANSYS Fluent. The interface tracking of the VOF model was carried out by GeoReconstruct scheme and the implicit body force formulation was activated to improve model stability under the gravity field imposed. Unsteady particle tracking was applied to the DPM particles. The different drag laws and bubble size model mentioned above were specially programmed and implemented as user defined functions.

The gas dispersion simulations were carried out with a transient solver at higher order discretization. For pressure discretization, RESTO! Scheme was used, and for continuity, momentum and turbulence equations the second order upwind scheme was used. For pressure-velocity coupling, the PISO scheme was used. The model was initialized with zero values for all variables, besides $k$ and $\varepsilon$ are guessed to be $k=0.01 \mathrm{~m}^{2} / \mathrm{s}^{2}$ and $\varepsilon=$ $0.001 \mathrm{~m}^{2} / \mathrm{s}^{3}$. The time-step was set to $0.01 \mathrm{~s}$ with the maximum number of 100 iterations per time-step. Simulations were performed for 20 seconds as same as the time duration of the gas release in the experiment.

\section{RESULTS AND DISCUSSION}

This section presents the predicted simulation results in comparison with experimental data presented by Engebretsen et al. [5], in addition to some references to the simulation results presented by Cloete et al. [4] and Bakli [8]. The calculations are stopped after 20 seconds, when quasi steady state is assumed. Fountain height, rise time and various velocity profiles are measured, compared, and presented in the following sections. The effect of drag model on subsea gas dispersion is evaluated based on fountain height, rise time and velocity predictions. Four different drag models are compared, namely spherical drag law, modified spherical drag law, Xia's drag law and Tomiyama's drag law. 


\subsection{Height of fountain and rising time}

The rising water is deflected outwards in a radial surface flow, and an elevation of the water surface occurs, due to momentum of the entrained water plume. The contour plot of volume fractions, showed in Fig. 3(a), illustrates the elevation of the water surface for basin experiment with gas flow rate $170 \mathrm{Nl} / \mathrm{s}$. The blue color indicates the water phase and red color indicates the atmospheric air. The yellow line represents the interface between the two phases, which is captured by the Geo Reconstruction Scheme. The fountain height was estimated by plotting the volume fractions against the position above the initial water surface (i.e. $7 \mathrm{~m}$ above the basin bottom), along the plume center line [8].

Fig. 3(b) is a vector plot of water velocities in the interaction zone, after quasi steady state conditions are reached. The water is radially deflected and the atmospheric air moves in the same direction, due to shear forces between water and atmospheric air.

The plume rise time is defined as the time to initial surface burst, i.e. the time it takes for the first bubbles to reach the water surface [8]. Table 1 presents a comparison of plume rising time and fountain height predicted by different drag models for experimental measurements at flow rate $170 \mathrm{Nl} / \mathrm{s}$ [14]. In the experiments, the initial fountain height when the plume reached the surface for the first time and the maximum fountain height were measured.

The spherical and modified spherical drag models predict almost identical results with respect to plume rising time and fountain height. However, initial and maximum fountain heights are under-predicted by both models. It should be noted that plume rising time predictions based on spherical and modified spherical drag models agree well with the experimental measurements. The plume rising time and initial fountain height predicted by Xia's drag model and Tomiyama's drag model are identical. However, Xia's drag model predicts slightly higher maximum fountain height compared to the Tomiyama's drag model. The rise time is over-predicted by both drag models similar to the results presented by Bakli [8]. Cloete et al. [4] presented a slightly lower simulated rise time compared to the current

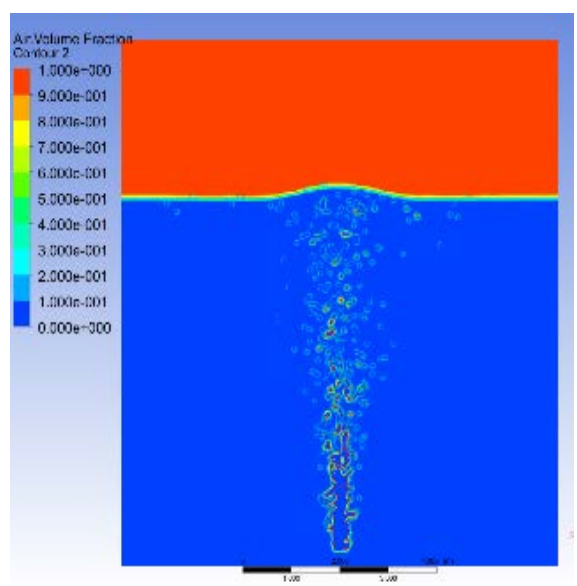

(a) Fountain

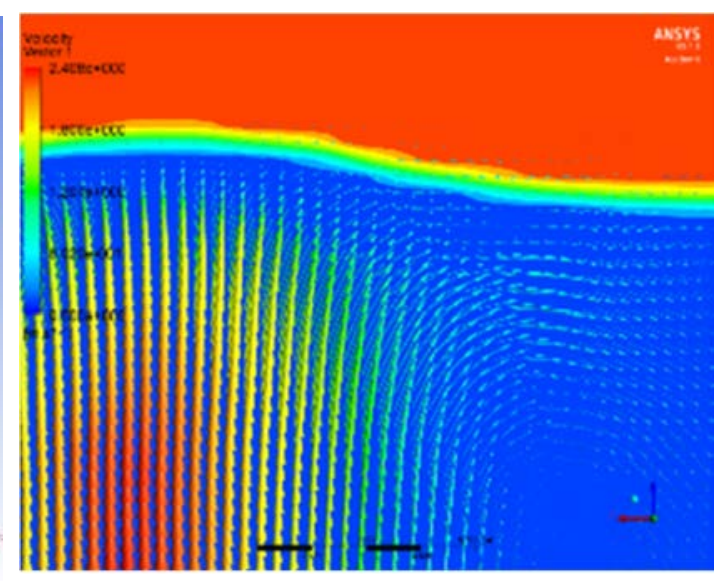

(b) Flow reflection at the surface

Figure 3: The bubble plume for basin experiment $170 \mathrm{Nl} / \mathrm{s}$. (a) Contour plot coloured by the volume fractions at the free water surface; (b) Velocity vector plot in the free surface region. 
Table 1: Comparison of plume rising time and fountain height predicted by different drag models for experimental measurements at flow rate $170 \mathrm{Nl} / \mathrm{s}$ [5].

\begin{tabular}{|l|c|c|c|c|c|}
\hline Parameter & Experiment & $\begin{array}{c}\text { Xia's } \\
\text { drag }\end{array}$ & $\begin{array}{c}\text { Spherical } \\
\text { drag }\end{array}$ & $\begin{array}{c}\text { Modified } \\
\text { spherical } \\
\text { drag }\end{array}$ & $\begin{array}{c}\text { Tomiyama's } \\
\text { drag }\end{array}$ \\
\hline Plume rising time (s) & 4.80 & 5.50 & 4.60 & 4.75 & 5.50 \\
\hline Initial fountain height (m) & 0.30 & 0.18 & 0.13 & 0.13 & 0.19 \\
\hline Maximum fountain height (m) & 0.65 & 0.43 & 0.34 & 0.34 & 0.35 \\
\hline
\end{tabular}

simulation results. Bakli [8] reported that initial values of turbulence parameters $(k-$ turbulent kinetic energy and $\varepsilon$ - turbulent dissipation rate) seem to have major effects on the bubble plume properties. These values refer to the initial turbulence level in the surrounding water, and the ratio between them may influence simulation results. This effect is further investigated by performing gas dispersion simulation with different initial turbulent kinetic energy values, $k=0.007 \mathrm{~m}^{2} / \mathrm{s}^{2}$ and $k=0.014 \mathrm{~m}^{2} / \mathrm{s}^{2}$. The initial turbulent dissipation rate is assumed to be constant $\varepsilon=0.001 \mathrm{~m}^{2} / \mathrm{s}^{3}$. It is observed that when turbulent kinetic energy $(k)$ is increased, relative to turbulent dissipation rate $(\varepsilon)$, the horizontal extension of the bubble plume may increase, which might contribute to a longer rise time. As shown in Fig. 4, significant difference of plume position is observed which is captured at 5 seconds. Hence it can be concluded that plume rising time is rather dependent on initial turbulence properties and drag models have minor impact on the predicted results.

As shown in Table 1, all the drag models under-predict the initial fountain height similar to the results presented by Cloete et al. [4]. Bakli [8] presents, on the other hand, a slightly over-predicted fountain height for the same experimental conditions. It should be noted that Xia's drag and Tomiyama's drag models that include bubble shape show better agreement than the ones without including bubble shape in general.

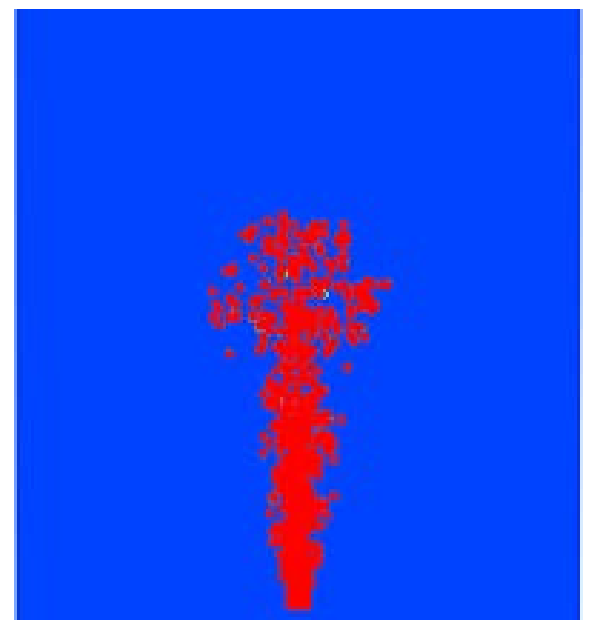

(a)

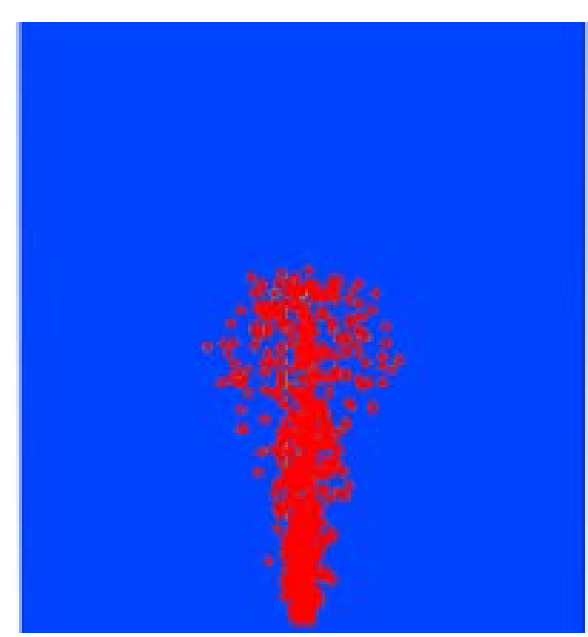

(b)

Figure 4: The bubble plume at 5 seconds. (a) $k=0.007 \mathrm{~m}^{2} / \mathrm{s}^{2}$; (b) $k=0.014 \mathrm{~m}^{2} / \mathrm{s}^{2}$. 


\subsection{Average plume velocity}

The velocity profiles predicted by different drag models for gas release rate $170 \mathrm{Nl} / \mathrm{s}$ are compared with the experimental data measured at two plume heights. As shown in Figs 5 and 6 , predicted velocity profiles based on spherical and the modified drag laws agree well with the experiment result, except for the center area of the plume. The model under-predicts the velocities at the center of the plume. It should be noted that the velocity measurements were performed using Höntzsch turbine flow meters [5]. These types of turbine flow meters are suited to measure mono-directional flow but may overestimate the vertical velocity component when the flows start to bend, when approaching the surface. This can be attributed to the deviations in measured and predicted velocities close to the plume center. It is also observed that spherical and modified spherical drag laws produce similar velocity predictions. However, spherical drag model produce slightly better velocity predictions compared to the modified spherical drag model within the range of radial position from 0 to $0.5 \mathrm{~m}$. The drag coefficients in spherical and modified spherical drag models are estimated over ranges of Reynolds numbers [13], [14]. The spherical drag model has better resolution in low Reynold's numbers compared to the modified spherical drag model. In addition, Reynolds numbers in spherical drag model is divided into seven ranges from $\mathrm{Re}=0$ to 10,000 while it is divided into five ranges in modified spherical drag model. This may be attributed to the better velocity predictions of spherical drag model within the range of radial position from 0 to $0.5 \mathrm{~m}$ where plume's Reynolds number is low.

Figs 7 and 8 show the experimental data compared with the current simulation results with Xia's and Tomiyama's drag models. The predicted results match the experimental data quite well at elevations $1.75 \mathrm{~m}$ and $3.80 \mathrm{~m}$. However, a slightly under prediction of the simulated velocities are observed right outside of the plume center similar to the results predicted by spherical and modified spherical drag models. It can be observed that the predicted results based on Tomiyama's drag model show a good comparison with experimental measurements compared to the other drag models even at the area close to the plume center. The predicted velocity data at elevation $3.80 \mathrm{~m}$ agree well with experimental data compared to the Xia's drag model. It is observed that the drag models (Xia's and Tomiyama's drag models) that include the shape of bubble give better velocity predictions. Hence, it can be concluded that bubble shape is important in under water gas dispersion mechanism. The Tomiyama's drag model includes both bubble shape and Reynolds number in the drag coefficient estimation. Hence, Tomiyama's drag model produce better velocity predictions compared to Xia's drag model where Reynolds number is not included in drag coefficient calculations.

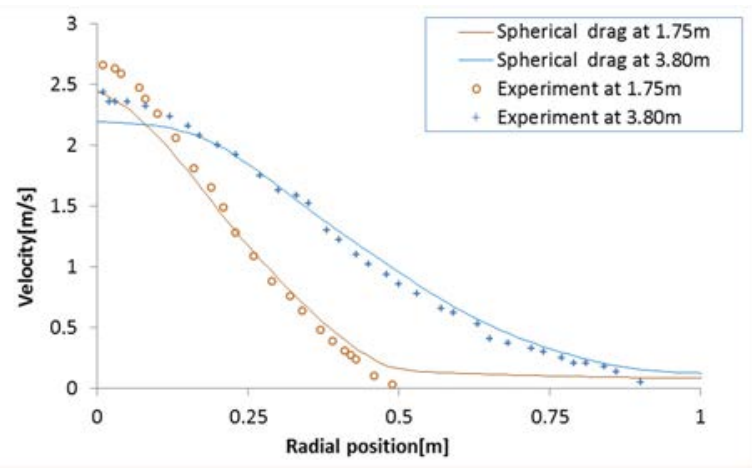

Figure 5: Velocity profiles at $z=1.75 m$ and $3.80 m-$ spherical drag law. 


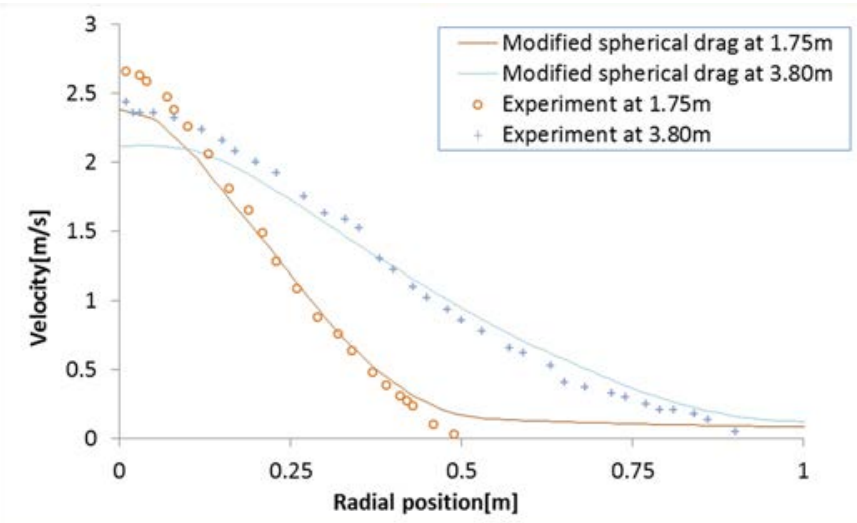

Figure 6: Velocity profiles at $z=1.75 m$ and $3.80 m$ - modified spherical drag law.

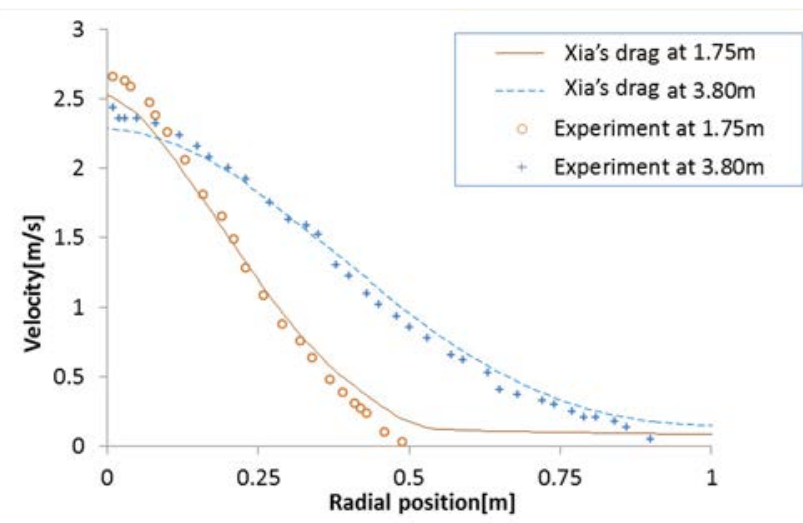

Figure 7: Velocity profiles at $z=1.75 m$ and $3.80 m-X i a$ 's drag law.

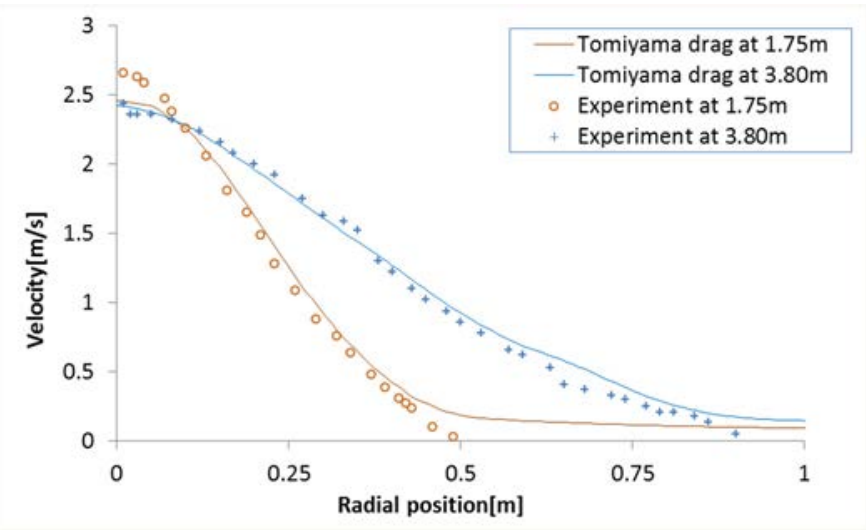

Figure 8: Velocity profiles at $z=1.75 m$ and $3.80 m$ - Tomiyama's drag law. 


\section{CONCLUSIONS}

This paper pres ents a comparis on between the different drag models developed in the literature and the effect of each one of them on the subsea gas dispersion. ANSYS Fluent is used as the CFD modelling framework of the subsea gas plume hydrodynamics, while the changes of bubble's density and size is incorporated as an external user defined functions (UDFs) hooked to the Fluent's main code structure. Four different drag models are compared, namely spherical drag law, modified spherical drag law, Xia's drag law and Tomiyama's drag law. The drag models are also included as external user defined functions (UDFs). A combination of the two methods; Eulerian-Eulerian and Lagrangian is used to model the bubbling behaviour of the subsea gas dispersion. The predicted results are validated against the experimental data presented by Engebretsen back in 1997. It is observed that the drag model in the CFD simulations seems to be a factor that could affect underwater plume physics. The predicted results by different drag models shows that the drag models including bubble shape show better agreement than the ones without including bubble shape in general. It can also be observed that velocity predictions based on Tomiyama's drag model show a good comparison with experimental measurements compared to the other drag models. The plume rising time is rather dependent on initial turbulence properties and drag models have minor impact on the predicted results. All the drag models under-predict the initial fountain height. However, Xia's drag and Tomiyama's drag models produce better initial fountain height predictions compared to the other drag models where bubble shape is not included in drag coefficient calculations.

\section{REFERENCES}

[1] Olsen, J.E. \& Skjetne, P., Current understanding of subsea gas release: a review. The Canadian Journal of Chemical Engineering, 94, pp. 209-219, 2016.

[2] Rew, P.G., Gallagher, P. \& Deaves, D.M., Dispersion of Subsea Releases: Review of Prediction Methodologies. Health and Safety Executive - Offshore Technology Report, OTH 95 465, 1995.

[3] Vollestad, P., Bubble plume modelling: including gas dissolution in an integral model, MSc thesis, Norwegian University of Science and Technology, 2013.

[4] Cloete, S., Olsen, J.E. \& Skjetne, P., CFD modelling of plume and free surface behaviour resulting from a sub-sea gas release. Applied Ocean Research, 31(3), pp. 220-225, 2009.

[5] Engebretsen T., Northug, T., Sjoen, K. \& Fannelop, T.K., Surface flow and gas dispersion from a subsea release of natural gas. Proceedings of the Seventh International Offshore and Polar Engineering Conference, Honolulu, USA, 1997.

[6] Pan, Q. \& Johansen, S.T., An enhanced k-epsilon model for bubble plumes. Proceedings of the Eighth International Conference on Multiphase Flow, 2013.

[7] Wu, K., Cunningham, S., Sivandran, S. \& Green, J., Modelling subsea gas releases and resulting gas plumes using Computational Fluid Dynamics. Journal of Loss Prevention in the Process Industries, 49, pp. 411-417, 2017.

[8] Bakli, M., Evaluation of gas and oil dispersion during subsea blowouts, MSc thesis, Department of Energy and Process Engineering, Norwegian University of Science and Technology (NTNU), 2014.

[9] Cloete, S.W.P., A mathematical modelling study of fluid flow and mixing in gas stirred ladles, Department of Process Engineering, Stellenbosch University, 2008.

[10] Skjetne, P. \& Olsen, J.E., A parcel based modelling concept for studying subsea gas release and the effect of gas dissolution. Progress in Computational Fluid Dynamics, 12(2/3), pp. 187-195, 2012. 
[11] Johansen, Ø., Rye, H., Melbye, A.G., Jensen, H.V., Serigstad, B. \& Knutsen, T., Deep Spill JIP - Experimental Discharges of Gas and Oil at Helland Hansen, SINTEF, Trondheim, 2000.

[12] Launder, B.E. \& Spalding, D.B., Lectures in Mathematical Models of Turbulence, London: Academic Press, 1972.

[13] Morsi, S.A. \& Alexander, A.J., An investigation of particle trajectories in two-phase flow systems. Journal of Fluid Mechanics, 55(2), pp. 193-208, 1972.

[14] Clift, R., Grace, J.R. \& Weber, M.E., Bubbles, Drops, and Particles, Courier Corporation, 2005.

[15] Xia, J.L., Ahokainen, T. \& Holappa, L., Analysis of flows in a ladle with gas-stirred melt. Scandinavian Journal of Metallurgy, 30(2), pp. 69-76, 2001.

[16] Takamasa, T. \& Tomiyama, A., Three-dimensional gas-liquid two-phase bubbly flow in a C-shaped tube. Proceedings of 9th International Topical Meeting on Nuclear Reactor Thermal Hydraulics (NURETH-9), San Francisco, CA, 1999.

[17] Laux, H. \& Johansen, S.T., A CFD analysis of the air entrainment rate due to a plunging steel jet combining mathematical models for dispersed and separated multiphase flows. Fluid Flow Phenomena in Metals Processing, Trondheim, 1999.

[18] Pan, Q., Modelling of turbulent flows with strong dispersed phase-continuous fluid interactions, NTNU, Trondheim, 2014. 